\title{
The AWE HPC Benchmark
}

\author{
Ron Bell \\ AWE plc \\ Aldermaston \\ Berkshire \\ RG7 4PR \\ ron.bell@awe.co.uk
}

\begin{abstract}
Near the end of 2005, AWE, Aldermaston placed an order for a Cray XT3 system with 3936 dual-core nodes (over 40 Tflops peak) to replace its existing HPC system. This paper describes the design of the benchmark used during the preceding competitive procurement including separate capacity and capability requirements analysis. Details are presented of the evaluation process and the relative results of the six HPC systems evaluated. These include the more than 2times speedup obtained by Cray by tuning the source code of the most important application.
\end{abstract}

